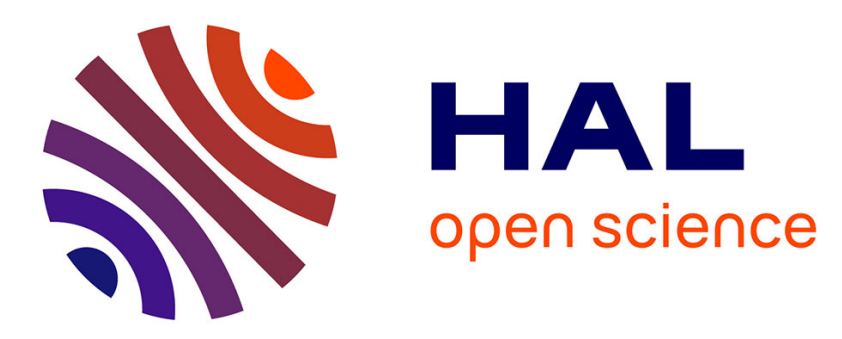

\title{
Visualization of an acoustic stationary wave by optical feedback interferometry
}

Patricio Fernando Urgiles Ortiz, Julien Perchoux, Antonio Luna Arriaga, Francis Jayat, Thierry Bosch

\section{To cite this version:}

Patricio Fernando Urgiles Ortiz, Julien Perchoux, Antonio Luna Arriaga, Francis Jayat, Thierry Bosch. Visualization of an acoustic stationary wave by optical feedback interferometry. Optical Engineering, 2018, 57 (05), pp.051502. 10.1117/1.OE.57.5.051502 . hal-01939780

HAL Id: hal-01939780

https://hal.laas.fr/hal-01939780

Submitted on 29 Nov 2018

HAL is a multi-disciplinary open access archive for the deposit and dissemination of scientific research documents, whether they are published or not. The documents may come from teaching and research institutions in France or abroad, or from public or private research centers.
L'archive ouverte pluridisciplinaire HAL, est destinée au dépôt et à la diffusion de documents scientifiques de niveau recherche, publiés ou non, émanant des établissements d'enseignement et de recherche français ou étrangers, des laboratoires publics ou privés. 


\title{
Visualization of an acoustic stationary wave by optical feedback interferometry
}

\author{
Fernando Urgiles $^{\mathrm{a}, \mathrm{b}}$, Julien Perchoux ${ }^{\mathrm{a},{ }^{*}, \text { Antonio Luna }}{ }^{\mathrm{a}}$, Francis Jayat $^{\mathrm{a}}$, Thierry Bosch ${ }^{\mathrm{a}}$ \\ ${ }^{a}$ LAAS-CNRS, Université de Toulouse, CNRS, INPT, Toulouse, France \\ ${ }^{b}$ Universidad Politécnica Salesiana Cuenca, Ecuador
}

\begin{abstract}
This paper presents a novel experimental technique for 2D imaging of dynamic acoustic pressure changes that is applied to visualize a stationary acoustic wave. This technique uses the optical feedback interferometry sensing scheme with a near infrared laser diode and a two-axis scanning system. The stationary acoustic wave is generated by using a $40 \mathrm{kHz}$ piezoelectric transducer pointing towards a concave acoustic reflector. The acoustic pressure dynamic changes are measured thanks to its impact on the propagating medium refractive index which variation is integrated along the laser optical path from the laser diode to a distant mirror and back. The imaging system records a 100x50 pixels image of the acoustic pressure in 66 minutes.
\end{abstract}

Keywords: Acoustic imaging, Optical Feedback Interferometry, Acousto-Optic sensing.

*Julien Perchoux, julien.perchoux @laas.fr

\section{Introduction}

The visualization of sound propagation has been a great challenge for researchers in recent years leading to the development of new systems to measure and reconstruct images of acoustic pressure waves. The tools that are normally used to detect and measure the acoustic field are microphones that have a high sensitivity but need to be inserted inside the acoustic field, which causes alterations in the measurements of complex fields and in the case of imaging an acoustic wave requires a matrix of several microphones. ${ }^{1}$ The detection of acoustic waves can be carried out by optical methods ${ }^{2-4}$ and particularly using interferometry based solutions without disturbing the pressure field. The weak changes of the refractive index in the transparent medium where the light propagates produce a modulation of the optical path along the trajectory of the laser beam. The phase shift induced by this modulation, that can be measured using interferometers, ${ }^{5-8}$ represents the integration of pressure changes over the laser trajectory.

However, the laser beam being of very small dimension with regards to the laser wavelength, producing an image of the acoustic waves requires to perform a raster scan so that the measurement at each position represent a pixel of the reconstructed image. While using traditional interferometric methods such as LDV, ${ }^{9}$ bulkiness of the optical systems and sensitivity to mechanical perturbations drastically limits the range of operation.

The method presented in this paper is based on Optical Feedback Interferometry (OFI), which is based on the beating effect between the emitted and the back-reflected wave from a distant reflector. OFI systems have previously been used to detect sound waves by monitoring the vibration of an exposed membrane ${ }^{10}$ and recently by sensing the acousto-optic effect. ${ }^{11}$ In this seminal work, Bertling et al have demonstrated the possibility for OFI sensors to image acoustic waves propagating in free-space. In the present work, we apply a similar method for the imaging of a stationary wave generated by coupling the acoustic source to a concave acoustic reflector. 


\section{Principle of operation}

An interferometric modulation of the laser power is generated when a phase-shifted portion of the laser beam is retro injected into the cavity from which it was originally emitted. ${ }^{12}$ While re-entering the laser cavity, the electric field of the back-scattered light interferes with the laser cavity inner field, which affects its phase and amplitude. The latest impacts directly the laser power emission which variations are measured by the laser in-package monitoring photodiode. The laser power fluctuations therefore provide an image of the information on the optical path variations that have followed the returning photons.

When applying OFI to the measurement of acoustic fields, one measures the changes in the optical path between the laser source and a distant mirror (as depicted in Fig. 1) that are due to the modulation of the refractive index induced by the pressure wave. Solving the laser rate equations under feedback conditions leads to the following expression of the laser emitted power $P_{F}::^{13}$

$$
P_{F}=P_{0}\left[1+m \cos \left(\omega_{F} \tau\right)\right],
$$

where $P_{0}$ is the laser power without optical feedback, $m$ is a modulation index that depends mainly on the reflectivity of the mirror, $\omega_{F}$ is the angular frequency of the lasers electric field and $\tau$ is the round-trip time of photons in the external cavity. In the case of pressure induced variation of the round-trip time $\tau$, this one can be described by $\tau=\tau_{0}+\delta \tau$ where $\tau_{0}$ is the round-trip time in absence of pressure change and $\delta \tau$ is the variation due to the pressure induced compression of the medium: ${ }^{11}$

$$
\delta \tau=\int_{0}^{L} \frac{2 \delta n(z)}{c} d z,
$$

with $\delta n$ the refractive index change over the external cavity length $L$ and $c$ the speed of light in vacuum.

Thus, with this technique, the acoustic pressure variations over an integration line are measured. In order to produce an image of the acoustic wave, it is required to move the sensor in each pixel position and to synchronize the signal acquisition in each position with the acoustic source excitation signal.

\section{Experimental setup}

The optical feedback interferometer consists in a distributed feedback laser diode ML725B11F with a transverse and longitudinal singlemode emission at $1310 \mathrm{~nm}$. The laser is beaming onto a silver mirror of diameter $6 \mathrm{~mm}$ through a collimating lens (Thorlabs C240TME-C). The external cavity length $\mathrm{L}$ between the laser and mirror is set to $130 \mathrm{~mm}$ and both are mounted on U-shaped rigid holder so that the optical path is not affected by any other perturbation than the acoustic wave propagation while performing the scan. The OFI set is associated to a translation system with two motorized linear stage of $100 \mathrm{~mm}$ travel range for the $\mathrm{X}$ axis and $50 \mathrm{~mm}$ travel range for the $\mathrm{Y}$ axis.

The acoustic source is an ultrasonic piezoelectric transmitter KPUS-40T-16T-K768 driven by a sinusoidal voltage of $10 \mathrm{~V}$ peak-to-peak amplitude at $40 \mathrm{kHz}$. Under these conditions, $30 \mathrm{~cm}$ away from the transducer, the acoustic pressure is expected to reach $117 \mathrm{~dB}$. It is displayed (see Fig. 1) so that the propagation axis is perpendicular to the optical propagation direction and facing a 


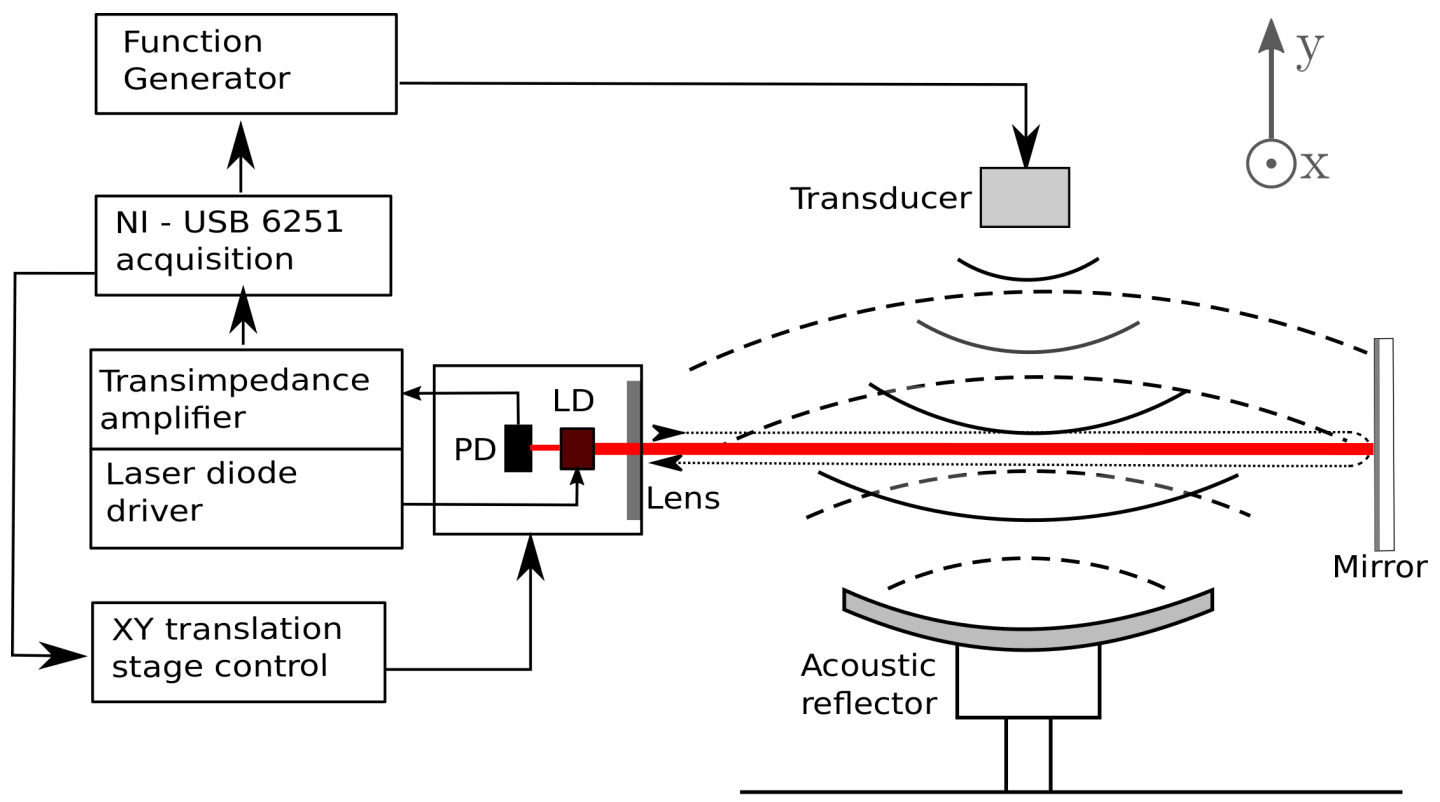

Fig 1 Schematic of the imager setup. The incident acoustic wave is represented in concentric solid line arcs, while the reflected acoustic is represented as dashed line arcs. LD and PD stand for laser diode and photodiode respectively.

parabolic reflector of $110 \mathrm{~mm}$ diameter leading to a focal distance of $40 \mathrm{~mm}$. The distance between the transducer and the reflector is $60 \mathrm{~mm}$ in order to obtain the stationary wave.

The amplitude of the signal variations are acquired through the in-package monitoring photodiode of the laser, which current is amplified by a custom made transimpedance amplifier then fed into a National Instrument acquisition card (NI-USB-6251) with a sampling frequency of $1 \mathrm{MHz}$. A Labview program controls the position of the linear translation stages in both axes (x,y), and at each pixel the photodiode amplified current is acquired as shown in the figure 2 in the case of pixel $(60,45)$, by windows of 1000 samples lenght. The acquisition of the signal at each pixel takes about $0.8 \mathrm{~s}$, and a time budget of $\mathrm{Y} \mathrm{s}$ is allowed to the displacement of the sensor and mechanical stabilization, all together leading to a global time of 66 minutes to perform the full image.

\section{Results and discussion}

Figures 2 and 3 present the sensor signal and its Fast Fourier Transform (FFT) respectively, for a given (x,y) position. In Fig. 3, the $40 \mathrm{kHz}$ peak amplitude has been demonstrated to be proportional to the acoustic field pressure. ${ }^{14}$

While saving the amplitude information of the FFT signal at $40 \mathrm{kHz}$ in each (x,y) position, the image of the acoustic pressure distribution in $(x, y)$ plane is reconstructed, showing a very clear behavior of a stationary wave. Fig. 4 shows this image in the case of a 100x50 mm with a resolution $1 \mathrm{~mm}$.

In addition, the image of the stationary wave can be plotted using the instantaneous amplitude of the signal. In order to improve the image quality, we applied a digital filter Pass-Band Butterworth 2 order with central frequency $40 \mathrm{kHz}$ and band-pass from $38-42 \mathrm{kHz}$ to each acquired 


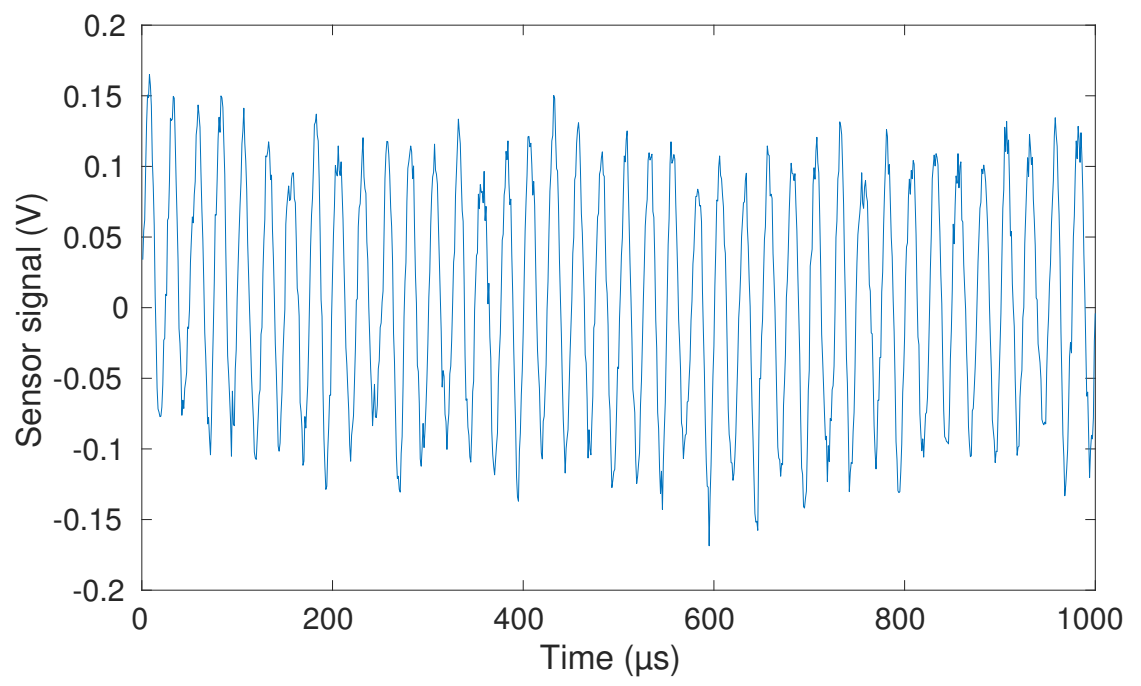

Fig 2 Time domain sensor signal acquisition for pixel $(60,45)$.

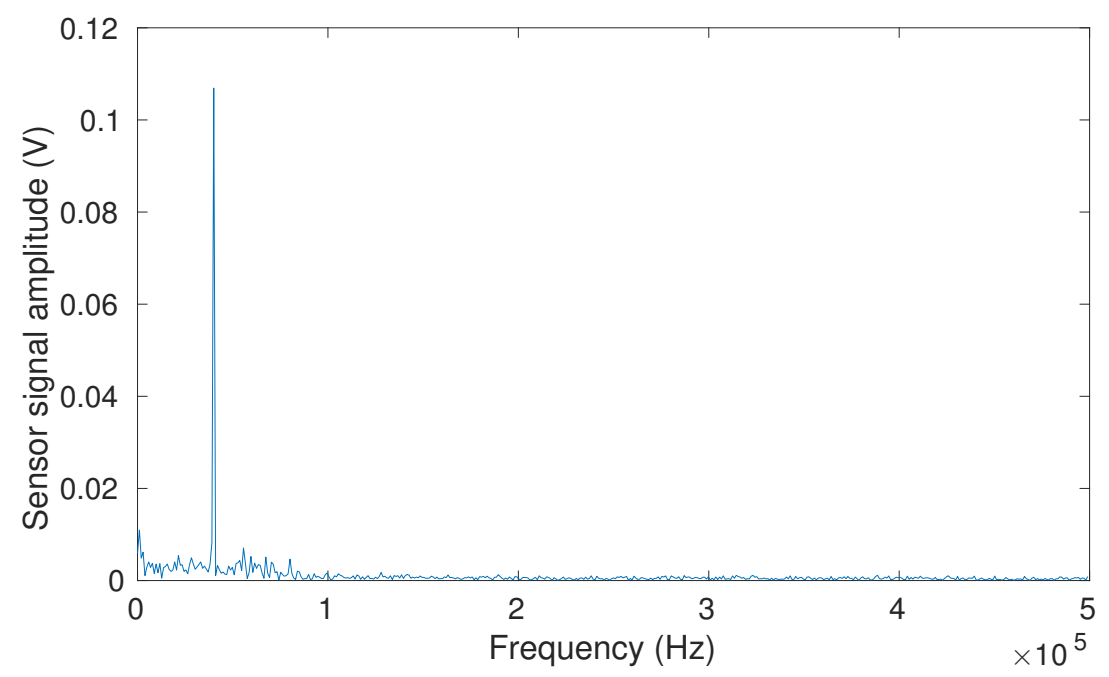

Fig 3 Fast Fourrier Transform of the signal plotted in Fig. 2 corresponding to pixel $(60,45)$.

signal. Figure 5 shows such a reconstructed image, while media 1 presents the evolution in time of this acoustic wave representation.

In order to clearly appreciate the position of the nodes and antinodes of the stationary wave, we have acquired and stored the time domain variations of the sensor signal over the 50 positions along the acoustic propagation axis. Figure 6 represents the sensor signals along the $\mathrm{x}$-axis at $\mathrm{y}=60 \mathrm{~mm}$ at 50 consecutive acquisition over a generator signal period. In this image, where the transducer location corresponds to the 50 abscissa, it is possible to observe the acoustic wavelength of $8 \mathrm{~mm}$ that corresponds to the frequency of $40 \mathrm{kHz}$ in the air. 


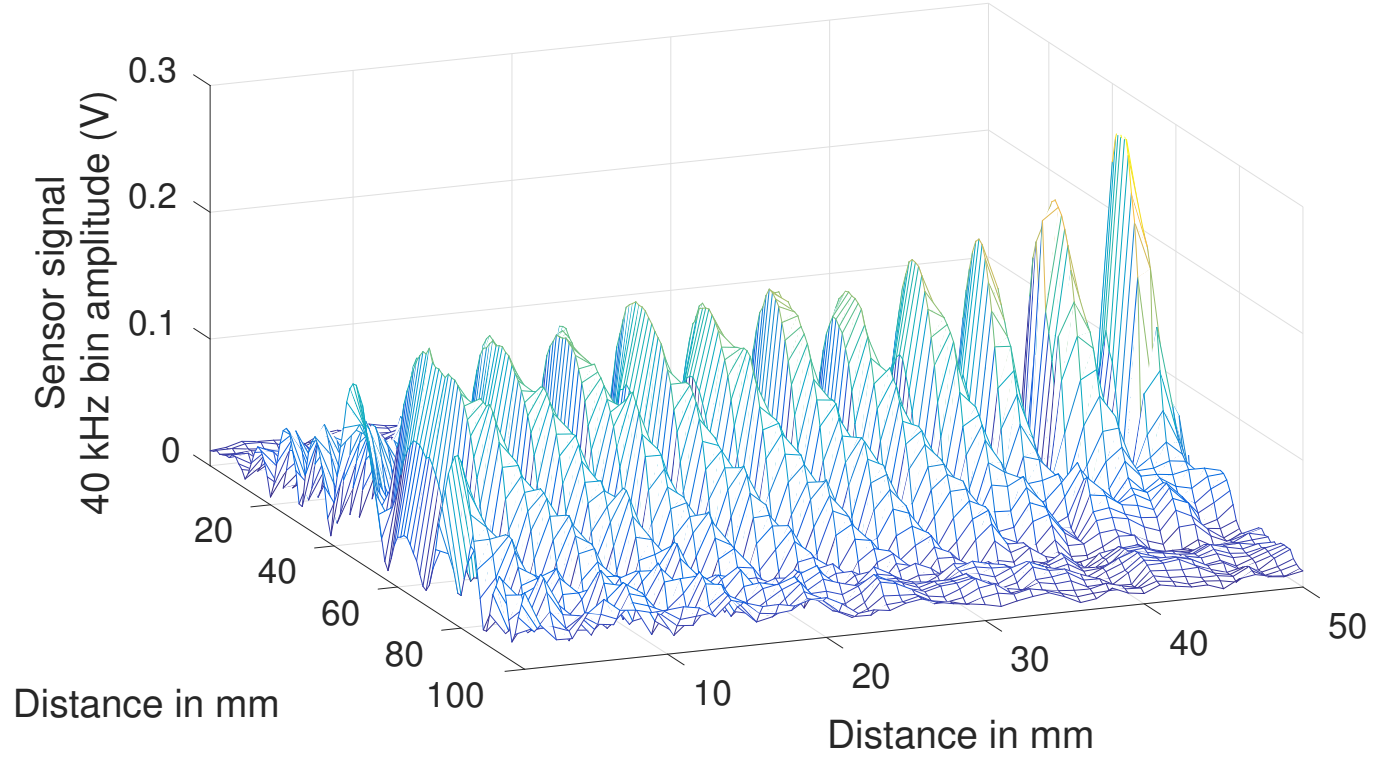

Fig 4 100x50 pixels image of the stationary pressure wave reconstructed from the $40 \mathrm{kHz}$ peak of the sensor signal spectrum at each pixel.

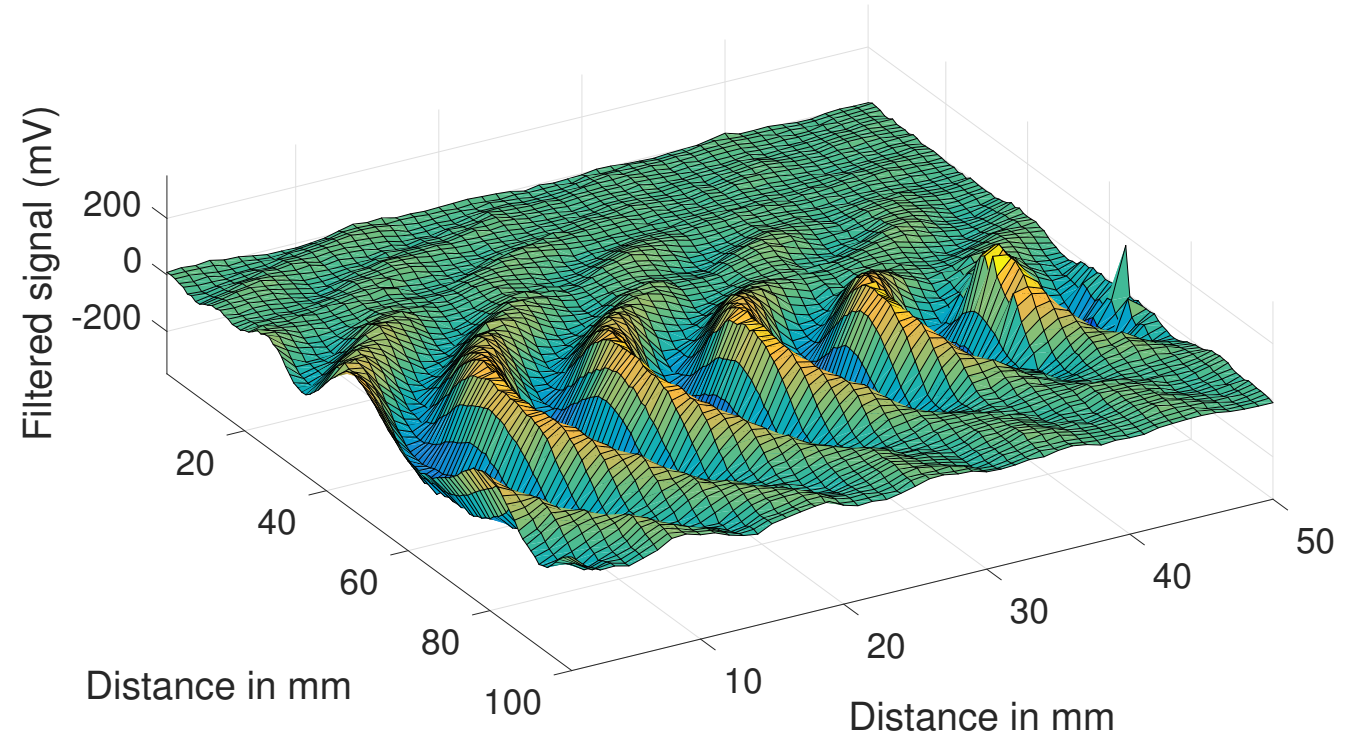

Fig 5 100x50 pixels instantaneous image of the stationary pressure wave after band-pass filtering of signals. Still image of from Video 1 (Video1, 5.8 MB) 


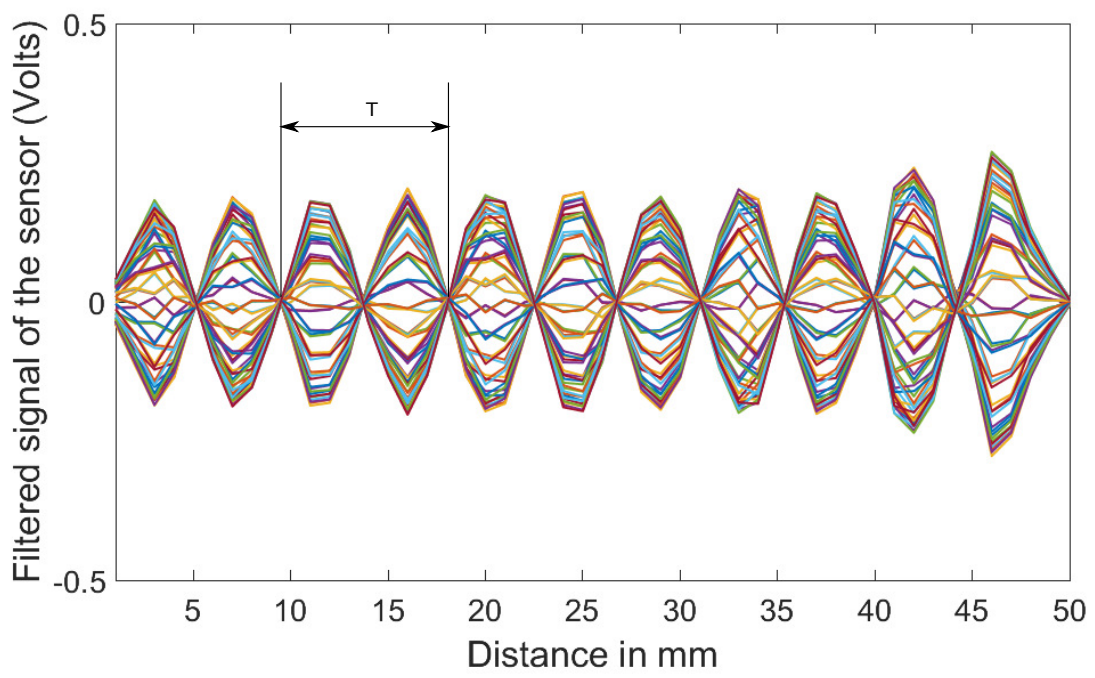

Fig 6 Acoustic propagation axis stored time domain signals showing nodes and anti-nodes of the pressure wave.

\section{Conclusion}

Thanks to the use of an imaging system based on OFI we demonstrated the visualization of pressure variations of an acoustic stationary wave produced by the reflection of the emitted wave by a concave reflector. The image that was produced has a resolution of $1 \mathrm{~mm}$ and it allows clear observation the nodes and anti-nodes positions. Further extensions of this work consist in observing acoustic wave shaping as for example focalisation by means of acoustic reflectors and improving the acquisition time of the imager with the objective to obtain the image in less than 66 minutes.

\section{Acknowledgments}

This work was supported by the Instituto de Fomento al Talento Humano of the Ecuadorian Government.

\section{References}

1 S. Siltanen, P. W. Robinson, J. Saarelma, et al., "Acoustic visualizations using surface mapping," The Journal of the Acoustical Society of America 135(6), EL344-EL349 (2014).

2 K. Hall, "Observing ultrasonic wave propagation by stroboscopic visualization methods," Ultrasonics 20(4), 159-167 (1982).

3 D. F. Comesaña, S. Steltenpool, G. Carrillo Pousa, et al., "Scan and paint: theory and practice of a sound field visualization method," ISRN Mechanical Engineering 2013 (2013).

4 N. Chitanont, K. Yaginuma, K. Yatabe, et al., "Visualization of sound field by means of schlieren method with spatio-temporal filtering," in Acoustics, Speech and Signal Processing (ICASSP), 2015 IEEE International Conference on, 509-513, IEEE (2015).

5 R. Malkin, T. Todd, and D. Robert, "A simple method for quantitative imaging of $2 \mathrm{~d}$ acoustic fields using refracto-vibrometry," Journal of Sound and Vibration 333(19), 4473 - 4482 (2014). 
6 Y. Oikawa, M. Goto, Y. Ikeda, et al., "Sound field measurements based on reconstruction from laser projections," in Acoustics, Speech, and Signal Processing, 2005. Proceedings.(ICASSP'05). IEEE International Conference on, 4, iv-661, IEEE (2005).

7 K. Ishikawa, K. Yatabe, N. Chitanont, et al., "High-speed imaging of sound using parallel phase-shifting interferometry," Optics express 24(12), 12922-12932 (2016).

8 L. Zipser, H. Franke, E. Olsson, et al., "Reconstructing two-dimensional acoustic object fields by use of digital phase conjugation of scanning laser vibrometry recordings," Applied optics 42(29), 5831-5838 (2003).

9 E. Olsson, N.-E. Molin, M. Sjodahl, et al., "Scattered ultrasound fields measured by scanning laser vibrometry," in Proceedings of SPIE, 5144, 717-727 (2003).

10 Y. Oikawa, T. Hasegawa, Y. Ouchi, et al., "Visualization of sound field and sound source vibration using laser measurement method," Proc. Int. Congr. Acoust.(ICA10) , 898 (2010).

11 K. Bertling, J. Perchoux, T. Taimre, et al., "Imaging of acoustic fields using optical feedback interferometry," Optics express 22(24), 30346-30356 (2014).

12 G. Giuliani, M. Norgia, S. Donati, et al., "Laser diode self-mixing technique for sensing applications," J. Opt. A : Pure Appl. Opt. 4, 283-294 (2002).

13 T. Bosch, C. Bes, L. Scalise, et al., "Optical feedback interferometry," Encyclopedia of Sensors X, 1-20 (2005).

14 F. Urgiles, J. Perchoux, and T. Bosch, "Characterization of acoustic sources by optical feedback interferometry," Proceedings 1(4) (2017).

Patricio Fernando Urgilés Ortiz was born in Cuenca-Ecuador on November 26, 1971. He completed his secondary studies in the Salesian Technical Superior Institute. He graduated from the Universidad Politecnica Salesiana as an Electronics Engineer in 2003. He obtained his specialist degree in University Teaching in 2005 in the Universidad del Azuay, in 2012 he received his Master's Degree in Electronic Engineering from the Pontificia Universidad Javeriana in Bogota Colombia. He is currently studying in the third year of the Doctoral program at the Institut National Polytechnique de Toulouse on the Topic of Acoustic images by Optical Feedback Interferometry.

Julien Perchoux was born in Vénissieux, France, in 1977. He received the B.Sc. degree in applied physics from the University of Montpellier, France, in 1999, the M.Sc.degree and the Ph.D. degree in microwave and photonics from the University of Toulouse - ISAE, in 2002 and 2005 respectively. He was graduated from the École Normale Supérieure, Cachan, France in 2001. $\mathrm{He}$ is with the Optoelectronics for Embedded System (OSE) team of the LAAS-CNRS (Toulouse, France) as an associated professor since 2005. His current research interests include semiconductor laser physics, biomedical imaging, flowmetry, acoustic imaging and design of embedded sensing systems.

Antonio Luna Arriaga received the M.S. degree in instrumentation from the INSA-Lyon, France in 2007. He completed his doctoral studies with the Group of Optoelectronics for Embedded Systems (LAAS-CNRS), INP-Toulouse, France in 2014. His current research focuses on real-time signal processing and architectures to enable the development of smart sensing devices.

Francis Jayat was born in Limoges in 1973. After studies at the University of Limoges and the Xlim laboratory in Electronics Microwaves optics and photonics, he is presently with the group of 
Optoelectronics for Embedded Systems of the LAAS-CNRS as an engineer dedicated to system automation and signal acquisition for optical sensors.

Thierry Bosch was born in 1965. He is presently a Full Professor at Toulouse INP and he is leading the group of Optoelectronics for Embedded Systems of the LAAS-CNRS. His scientific interests are related to laser industrial instrumentation development including range finding techniques, vibration and velocity measurements. He has co-authored around 50 papers in archival journals and he has been invited to author the chapter dedicated to self-mixing sensors published in the Encyclopedia of Sensors. He has been Guest co-Editor for Journal of Optics (June 1998 and November 2002), Optical Engineering (January 2001) and Sensor (March 2017) on laser Sensors for Displacement, Distance, Flow, Velocity and Vibrations Measurements. In 2011 and 2014, he was chairing the special session on self-mixing during the IEEE Sensors $10 \& 13$ th Annual Conference. He won the Mechatronics Award (Research Category) during the European Mechatronics Meeting in 2010 and the Jean Ebbeni Price from the CMOI Technical Committee of the French Optical Society in 2011.

\section{List of Figures}

1 Schematic of the imager setup. The incident acoustic wave is represented in concentric solid line arcs, while the reflected acoustic is represented as dashed line arcs. LD and PD stand for laser diode and photodiode respectively.

2 Time domain sensor signal acquisition for pixel $(60,45)$.

3 Fast Fourrier Transform of the signal plotted in Fig. 2 corresponding to pixel $(60,45)$.

$4100 \times 50$ pixels image of the stationary pressure wave reconstructed from the $40 \mathrm{kHz}$ peak of the sensor signal spectrum at each pixel.

$5100 \times 50$ pixels instantaneous image of the stationary pressure wave after band-pass filtering of signals. Still image of from Video 1 (Video1, 5.8 MB)

6 Acoustic propagation axis stored time domain signals showing nodes and antinodes of the pressure wave. 